\title{
Qualidade de vida e incapacidade relacionada à saúde de crianças com migrânea
}

\author{
Gabriela Natália Ferracini', Fabíola Dach², José Geraldo Speciali \\ 'Fisioterapeuta, doutoranda em Neurociências da Faculdade de Medicina de \\ Ribeirão Preto (FMRP-USP), Ribeirão Preto, SP, Brasil \\ ${ }^{2}$ Doutora em Neurologia, Médica assistente do Ambulatório de Cefaleia do Hospital das Clínicas de \\ Ribeirão Preto (FMRP-USP), Ribeirão Preto, SP, Brasil \\ ${ }^{3}$ Professor associado de Neurologia do Departamento de Neurociências e Ciências do \\ Comportamento da Faculdade de Medicina de Ribeirão Preto (FMRP-USP), Ribeirão Preto, SP, Brasil \\ Ferracini GN, Dach F, Speciali JG. Qualidade de vida e incapacidade relacionada à \\ saúde de crianças com migrânea. Headache Medicine. 2012;3(4):203-5
}

\section{RESUMO}

A migrânea leva a uma redução da participação de crianças nas atividades de casa e de lazer e no rendimento escolar, além de comprometer os aspectos psicológicos, gerando um impacto negativo na qualidade de vida relacionada à saúde. Este estudo objetivou avaliar a incapacidade que esta condição causa na vida diária de crianças de 6 a 12 anos de idade de ambos os gêneros e se influencia a qualidade de vida. A amostra foi composta por 50 crianças com migrânea sem aura e 50 crianças sem histórico de cefaleia. Para verificar a incapacidade aplicou-se o questionário Pediatric Migraine Disability Score e para qualidade de vida o questionário Pediatric Quality of Life Inventory 4.0 ${ }^{\mathrm{TM}}$. Os meninos perderam em média 13 (DP 26,36 ) dias e as meninas em média 15 (DP 22,43) dias sobre atividade e rendimento escolar, tarefas de casa e lazer, em três meses. De acordo com o grau de incapacidade, 19 (38\%) apresentaram pouca ou ausência de incapacidade (Grau I), 17 (34\%) leve (Grau II), sete (14\%) moderada (Grau III) e sete (14\%) incapacidade grave (Grau IV) sobre atividade e rendimento escolar, tarefas de casa e lazer. A qualidade de vida, pela percepção dos pais, é pior nas crianças com migrânea [IC95\% $(-16,92--6,18)$ p <0,01], mas na percepção das crianças é semelhante nos dois grupos. Quando se analisam separadamente os vários domínios do questionário, pela percepção dos pais, há diferenças nos domínios físico [IC95\% $(-15,24--1,88)$ $p<0,01]$ e psicossocial [IC95\% $(-17,96--6,74) p<0,01]$. Enquanto que pela autoavaliação das crianças, houve diferença nos domínios emocional [IC95\% $(-16,95--5,70)$ $p<0,01]$ e escolar [IC95\% $(-15,90--5,74) p<0,01]$. Não foi encontrada correlação entre os dias perdidos devido à migrânea e a qualidade de vida.

Palavras-chave: Cefaleia; Crianças; Qualidade de vida

\section{INTRODUÇÃO}

Cefaleia é a causa mais comum de dor na infância, com alto risco para tornar-se crônica e persistente na idade adulta.(1) $A$ incapacidade que a migrânea causa durante e entre as crises leva a uma redução da participação da criança nas atividades familiar, social e 
escolar, além do comprometimento dos aspectos psicológicos, gerando um impacto negativo na qualidade de vida. ${ }^{(2)}$

A Qualidade de Vida relacionada à saúde (QVRS) compreende os efeitos que as doenças e os tratamentos têm sobre a vida diária e a satisfação pessoal.(3) $\bigcirc$ impacto sobre a QVRS pode ser avaliado por meio de instrumentos multidimensionais, que mensuram a percepção subjetiva e individual (autorrelato) nos aspectos físico, emocional, psicológico e social. ${ }^{(3)} \cup m$ instrumento genérico, utilizado para a população pediátrica e com validação para a população brasileira ${ }^{(4)}$ é o Pediatric Quality of Life Inventory (PedsQL ${ }^{\text {TM }} 4.0$ ), (5) que analisa a percepção da criança e de seus pais perante a doença. Além disso, há um instrumento mais específico para se mensurar a incapacidade que a migrânea causa na vida de crianças denominado Pediatric Migraine Disability Score (PedMIDAS), ${ }^{(6)}$ desenvolvido por meio da versão utilizada em adultos, o Migraine Disability Assessement Score. ${ }^{(7)}$

\section{OBJETIVO}

Avaliar a incapacidade que a migrânea causa na vida diária de crianças de 6 a 12 anos de idade de ambos os gêneros e se ela influencia a qualidade de vida.

\section{METODOLOGIA}

A amostra foi composta por 100 crianças 168 meninas e 32 meninos) de 6 a 12 anos de idade (média 9,9 anos, DP 1,6), divididas em dois grupos: grupo de estudo composto por crianças com diagnóstico de migrânea sem aura de acordo com a segunda edição da Classificação Internacional de Cefaleias, ${ }^{(8)}$ do ambulatório de Cefaleia e Algias Craniofaciais da Infância do Hospital das Clínicas da Faculdade de Medicina de Ribeirão Preto; grupo controle composto por crianças sem histórico de cefaleia há pelo menos seis meses, selecionadas do Ambulatório de Problemas de Crescimento e Desenvolvimento. Foram excluídas as crianças que apresentassem doenças musculares, dermatológicas, neurológicas graves e inflamatórias crônicas, as que não compreendessem o que era explicitado ou as que não concordassem em participar do estudo. $\bigcirc$ consentimento foi dado pelos pais ou responsáveis, com assinatura de um termo de consentimento livre e esclarecido. Para verificar a incapacidade, foi aplicado o questio- nário Pediatric Migraine Disability Score ${ }^{(6)}$ e, para qualidade de vida, o questionário Pediatric Quality of Life Inventory 4.0 $0^{\text {TM }}{ }^{(5)}$

\section{RESULTADOS}

Os meninos perderam em média 13 (DP 26,36) dias e as meninas em média 15 dias (DP 22,43) sobre atividade e rendimento escolar, tarefas de casa e lazer, em três meses, não havendo diferença significativa entre os gêneros [IC95\% $(-14,02-16,67) ; p=0,86]$.

De acordo com o grau de incapacidade, 19 (38\%) crianças apresentaram pouca ou ausência de incapacidade (Grau I), 17 (34\%) incapacidade leve (Grau II), sete (14\%) moderada (Grau III) e sete (14\%) incapacidade grave (Grau IV) sobre atividade e rendimento escolar, tarefas de casa e lazer. Na análise em separado de meninos e meninas, $40 \%$ das meninas apresentaram pouca ou ausência de incapacidade (Grau I), 29\% incapacidade leve (Grau II), 17\% moderada (Grau III) e $14 \%$ grave (Grau IV). Os meninos apresentaram 32\% pouca ou ausência de incapacidade (Grau I), 50\% leve (Grau II), 12\% moderada (Grau III) e 6\% grave (Grau IV) (Figura 1).

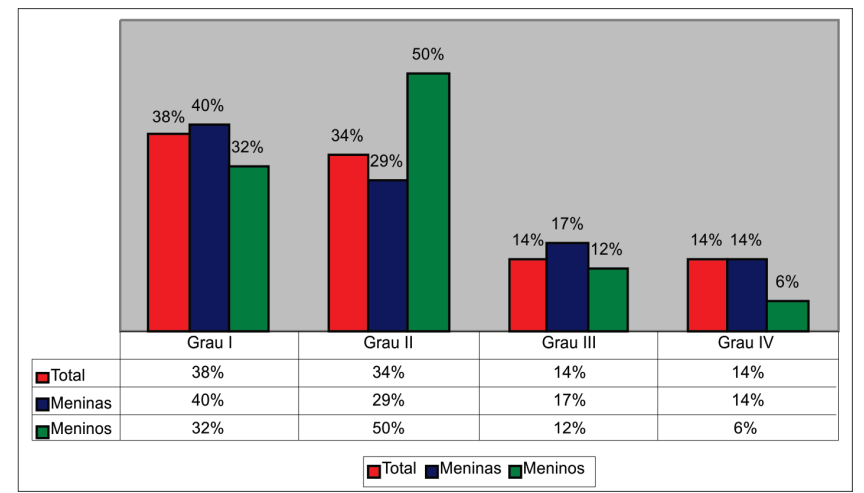

Figura 1 - Graus de incapacidade do PedMIDAS entre os gêneros do Grupo estudo.

A qualidade de vida, pela percepção dos pais, é pior nas crianças com migrânea [IC95\% (-16,92 - -6,18) $p<0,01]$, mas na percepção das crianças a qualidade de vida é semelhante nos dois grupos. Quando se analisa separadamente os domínios do questionário, pela percepção dos pais, há diferenças nos domínios físico [IC95\% (-15,24 - - 1,88), $p<0,01]$ e psicossocial [IC95\% $(-17,96--6,74), p<0,01]$. Enquanto que pela autoavaliação das crianças, houve diferença nos domínios emocional [IC95\% $(-16,95--5,70) p<0,01]$ e escolar [IC95\% (-15,90 - -5,74) $p<0,01]$ (Tabela 1). 


\begin{tabular}{|c|c|c|c|c|c|c|c|c|}
\hline \multirow[b]{3}{*}{ Escalas } & \multirow[b]{3}{*}{ Número de itens } & \multicolumn{7}{|c|}{ Grupo } \\
\hline & & \multicolumn{3}{|c|}{ Estudo } & \multicolumn{4}{|c|}{ Controle } \\
\hline & & $\mathrm{N}$ & Média & DP & $n$ & Média & DP & Valor $p$ \\
\hline \multicolumn{9}{|l|}{ Avaliação das crianças } \\
\hline Escore total & 23 & 50 & 61,45 & 12,48 & 50 & 64,53 & 10,83 & 0,18 \\
\hline Saúde física & 8 & 50 & 69,73 & 12,48 & 50 & 70,27 & 15,48 & 0,17 \\
\hline Social & 5 & 50 & 80,00 & 20,53 & 50 & 80,00 & 21,10 & 0,72 \\
\hline Saúde emocional & 5 & 50 & 55,00 & 20,20 & 50 & 60,00 & 20,53 & $<0,01^{*}$ \\
\hline Escolar & 5 & 50 & 50,00 & 18,84 & 50 & 52,50 & 18,67 & $<0,01^{*}$ \\
\hline Saúde Psicossocial & 15 & 50 & 68,07 & 17,66 & 50 & 58,07 & 16,66 & 0,07 \\
\hline \multicolumn{9}{|l|}{ Avaliação dos pais } \\
\hline Escore total & 23 & 50 & 57,50 & 13,42 & 50 & 69,53 & 9,89 & $<0,01^{*}$ \\
\hline Saúde física & 8 & 50 & 67,25 & 16,93 & 50 & 75,81 & 14,28 & $<0,01^{*}$ \\
\hline Social & 5 & 50 & 80,00 & 17,22 & 50 & 80,00 & 22,41 & 0,63 \\
\hline Saúde emocional & 5 & 50 & 50,00 & 24,81 & 50 & 55,00 & 21,10 & 0,06 \\
\hline Escolar & 5 & 50 & 50,00 & 18,66 & 50 & 55,00 & 18,69 & 0,18 \\
\hline Saúde Psicossocial & 15 & 50 & 53,57 & 15,37 & 50 & 65,92 & 10,50 & $<0,01^{*}$ \\
\hline
\end{tabular}

n: número da amostra; DP: desvio-padrão. * $p<0,05$

Não foi encontrada correlação entre os dias perdidos devido à cefaleia e a qualidade de vida.

\section{CONCLUSÃO}

O grau de incapacidade foi em geral ausente ou leve em $72 \%$ das crianças, e apenas $14 \%$ apresentaram o grau de incapacidade grave.

A qualidade de vida não se apresentou pior em crianças com migrânea, pela percepção das crianças, mas pela percepção dos pais, a qualidade de vida relacionada á saúde é pior em crianças com migrânea.

A incapacidade que a migrânea causa nas atividades diárias não parece interferir na qualidade de vida.

\section{REFERÊNCIAS}

1. Abu-arafeh I, Razak S, Sivaraman B, Graham C. Prevalence of headache and migraine in children and adolescents: a systematic review of population-based studies. Dev Med Child Neurol 2010;52(12):1088-97.

2. Isigkeit AR, Thyen U, Stöven H, Schwarzenberger J, Schmucker P. Pain among children and adolescents: Restrictions in daily living and triggering factors. Pediatrics. 2005; 1 15(2): e152-e162.

3. The Whogol Group. The development of the World Health Organization quality of life assessment instrument (the
WHOQOL). In: Orley J, Kuyken W editors. Quality of life assessment: international perspectives. Heidelberg: Springer Verlag 1994 p. 41-60.

4. Klatchoian DA, Len CA, Terreri MTRA, Silva M, Itamoto C, Ciconelli RM, et al. Qualidade de vida de crianças e adolescentes de São Paulo: confiabilidade e validade da versão brasileira do questionário genérico Pediatric Quality of Life InventoryTM versão 4.0. Jornal de Pediatria. 2008; 24(4): 308-15.

5. Varni Jw, Seid M, Kurti PS. PedsQI 4.0: reliability and validity of the Pediatric Quality of Life InventoryTM Version 4.0 generic core scales in healthy and patient populations. Med Care. 2001;39: 800-12.

6. Hershey Ad, Power Sw, Vockell Al, Lecates SI, Kabbouche Ma, Maynard MK. PedMIDAS: development os a questionnaire to assess disability of migraines in children. Neurology. 2001; 57: 2034-9.

7. Stewart Wf, Lipton R, Dowson Ai, Sawyer J. Development and testing of the migraine disablity; The Migraine Disability Assessment (MIDAS) Questionnaire to assess headache related disability. Neurology, 2001;56 (6 Suppl): S20-8.

8. Headache Classification Subcommitte Of The International Headache Society. The International Classifications of Headache Disordes. 2 ed. Cephalalgia. 2004 ;24 (1): 9-160.

Este estudo foi aprovado pelo Comitê de Ética em Pesquisa do Hospital das Clínicas da Faculdade de Medicina de Ribeirão Preto da Universidade de São Paulo - HCFMRP/USP, sob protocolo 3694/2010. 